\title{
Wogonin inhibits osteoclast differentiation by inhibiting NFATc1 translocation into the nucleus
}

\author{
XIAOLIN GENG, LIBIN YANG, CHAO ZHANG, HUA QIN and QIUDONG LIANG \\ Department of Orthopedic Surgery I, The First Affiliated Hospital of Xinxiang Medical University, \\ Weihui, Henan 453100, P.R. China
}

Received August 25, 2014; Accepted June 2, 2015

DOI: 10.3892/etm.2015.2615

\begin{abstract}
The aim of the present study was to identify a natural product with the ability to inhibit nuclear factor of activated T cells c1 (NFATc1) translocation from the cytoplasm to the nucleus by high-throughput screening, and to investigate the effect of the natural product upon osteoclast differentiation and its underlying mechanism. An NFATc1 antagonist redistribution assay was performed in U2OS-NFATc1 cells against a natural product library, and Wogonin was found to have the ability to inhibit the NFATc1 translocation from the cytoplasm to the nucleus. The effect of Wogonin on NFATc1 transcription activation was further determined by luciferase assay. An osteoclast differentiation assay was executed to evaluate the effect of Wogonin on osteoclast differentiation. The effect of Wogonin upon the vital genes in osteoclast differentiation was investigated using fluorescent quantitative polymerase chain reaction analysis. The natural product Wogonin significantly inhibited the translocation of NFATc1 from the cytoplasm to the nucleus and its transcriptional activation activity. Wogonin also significantly inhibited osteoclast differentiation and decreased the transcription of osteoclast-associated immunoglobulin-like receptor, tartrate-resistant acid phosphatase and calcitonin receptor. In conclusion, the natural product Wogonin inhibited osteoclast differentiation through the inhibition of NFATc1 translocation from the cytoplasm to the nucleus, and thus the downregulation of genes associated with osteoclast differentiation, which marked Wogonin as a potential treatment for osteoporosis.
\end{abstract}

\section{Introduction}

Osteoporosis, a systemic skeletal disease, is highly relevant to age and is characterized by reduced bone mass, decreased bone mineral composition and bone mineral/matrix ratio,

Correspondence to: Dr Qiudong Liang, Department of Orthopedic Surgery I, The First Affiliated Hospital of Xinxiang Medical University, 88 Jiankang Road, Weihui, Henan 453100, P.R. China E-mail: qiudongliangen@163.com

Key words: osteoporosis, osteoclast, NFATc1, Wogonin bone thinning and bone microstructure degeneration, including reduced trabecular bone, resulting in increased bone brittleness and bones prone to fracture. Osteoporosis occurs mainly due to the enhancement of bone resorption, decreased bone formation or both, with various causes. Bone marrow density, bone remodeling and bone integrity are regulated by osteoblasts and osteoclasts (1). In normal conditions, these two types of cells are in a state of equilibrium to ensure the normal development of the human skeleton and homeostasis of bone formation. Enhanced osteoclast activity or decreased osteoblast activity can break the balance, leading to osteoporosis with reduced bone mass and pathological bone-thinning changes (2); therefore, inhibiting the activity of osteoclasts by inhibiting osteoclast differentiation is an important strategy for treating osteoporosis.

Nuclear factor of activated T cells (NFAT) proteins are a group of proteins that exhibit transcriptional activity and are found universally in animals. NFATs can regulate cell proliferation and differentiation at the transcriptional level (3). There are five major members of the NFAT family: NFATc1, NFATc2, NFATc3, NFATc4 and NFAT5. Among them, NFATc1 comprises a group of transcriptional factors that are regulated by calcineurin and may affect the signaling of T cells and tissue development. The inactivated form of NFATc1 is located in the cytoplasm and does not have transcriptional activity. The activated NFATc1, which exhibits transcriptional activity, can be translocated into the nucleus where it regulates the expression of the downstream genes (4). A previous study found that the specific activation of NFATc1 could induce the differentiation of osteoclast precursors into mature osteoclasts, whereas inhibiting the activity of NFATc1 can inhibit osteoclast differentiation (5). It has also been found that numerous proteins that are vital for osteoclast differentiation, including tartrate-resistant acid phosphatase (TRAP), calcitonin receptor and osteoclast-associated immunoglobulin-like receptor (OSCAR) are regulated by NFATc1 (6-8). NFATc1 may, therefore, become an important therapeutic target for treating osteoporosis.

Currently, drugs for osteoporosis contain substances including estrogen, vitamin $\mathrm{D}$, calcitonin and bisphosphonates. Biophosphonates are the most commonly used drugs for treating osteoporosis, with the main mechanism being osteoclast inhibition; however, the long-term use of bisphosphonates can have adverse effects. Finding novel therapeutic 
targets for the inhibition of osteoclasts is therefore important for the treatment of osteoporosis.

The aim of the present study was to use a high-throughout screening system to find a natural product that could clearly inhibit NFATc1 translocation into the nucleus. Among the potential natural products was Wogonin, which comes from the Traditional Chinese Medicine herb, Scutellaria baicalensis; however, recent research into Scutellaria baicalensis has mainly focused on its anti-tumor activity, with one study finding that Wogonin functions as an anti-colon cancer agent by regulating the $\mathrm{Wnt} / \beta$-catenin pathway $(9,10)$. To date, however, there have been no studies regarding the mechanism underlying the inhibitory effect of Wogonin on NFATc1 and osteoclasts. The effect of Wogonin on the inhibition of osteoclast differentiation was, therefore, also investigated.

\section{Materials and methods}

Cell culture. Mouse mononuclear macrophage (RAW264.7) cells (American Type Culture Collection, Manassas, VA, USA) were cultured in Dulbecco's modified Eagle's medium (DMEM; Sigma, St. Louis, MO, USA) with $10 \%$ fetal bovine serum (FBS; Sigma), $100 \mathrm{U} / \mathrm{ml}$ penicillin and $100 \mathrm{mg} / \mathrm{ml}$ streptomycin. Cells were incubated in $5 \% \mathrm{CO}_{2}$ at $37^{\circ} \mathrm{C}$. U2OS-EGFP-NFATc1 cells (Bio-Images, Glasgow, UK) were cultured in DMEM with $10 \% \mathrm{FBS}, 100 \mathrm{U} / \mathrm{ml}$ penicillin, $100 \mathrm{mg} / \mathrm{ml}$ streptomycin and $0.5 \mathrm{mg} / \mathrm{ml} \mathrm{G} 418$.

Osteoclast differentiation. RAW264.7 cells were seeded onto a 24-well plate with a density of $2 \times 10^{4}$ cells/well and incubated with DMEM until the cells were $70 \%$ confluent. The cells were washed with fresh medium, and $100 \mathrm{ng} / \mathrm{ml}$ receptor activator of nuclear factor $\kappa \mathrm{B}$ ligand (RANKL; Sigma) was added into the medium, and the cells were cultured for 3 days for differentiation. The cells were washed with fresh medium, and cultured in medium with $100 \mathrm{ng} / \mathrm{ml}$ RANKL and a corresponding compound for testing for 2 days. The amount of TRAP in the cell was detected in accordance with the instructions of the TRAP detection kit (Beyotime Institute of Biotechnology, Suzhou, China) in order to determine the effect of the compound on osteoclast differentiation. The specific method was as follows: The medium was removed and each well of the plate was washed by phosphate-buffered saline (PBS) once; $50 \mathrm{ml}$ fixing solution was added into each well, and the cells were fixed at room temperature for $5 \mathrm{~min}$. The cells were then washed 3 times and $50 \mathrm{ml}$ TRAP chromogenic substrate was added and incubated at $37^{\circ} \mathrm{C}$ for $20-60 \mathrm{~min}$. Osteoclasts were stained in red, and the degree of osteoclast differentiation was observed under the microscope (X80; Olympus Corporation, Tokyo, Japan; magnification, x200).

Detection of NFATcl within and outside of the nucleus. U2OS cells were stably transfected with EGFP-NFATc1 and cultured until the cells grew confluently. Subculture was performed at a ratio of 1:3 in a 96-well IN Cell ${ }^{\mathrm{TM}}$ Analyzer 1000 (GE Healthcare Life Sciences, Little Chalfont, UK) and the cells were cultured overnight. The next day, the natural product for detection (130 single and small molecules in total, extracted from Chinese medicine sources; Selleckchem, Houston, TX, USA) or the solvent (control group) was added. In addition,
RANKL was added (100 ng/ml), and the mixture was incubated for $24 \mathrm{~h}$. Following incubation, the cells were washed twice with PBS, and then $2 \mathrm{mM}$ Hoechst 33342 was added to stain the nucleus for 15 min. Finally, the IN Cell Analyzer 1000 was used to capture video images and software was used to analyze the videos. Three repeatable wells were established for each treatment and six views were captured for each well. The ratio of the fluorescent intensity of the nucleus to the cytoplasm was used to detect the translocation of NFATc1 into the nucleus.

Quantitative polymerase chain reaction (qPCR). RAW264.7 cells were cultured in a six-well plate until the cells were $60 \%$ confluent. RANKL (100 ng/ml) and different concentrations (5.0, 1.0, 0.1 and $0.0 \mathrm{mM}$ ) of Wogonin (Selleckchem) were added. Dimethyl sulfoxide (DMSO) was used as a control. The cells were incubated for $24 \mathrm{~h}$ and then the total mRNA was extracted. The specific method was as follows: Cells were washed three times with PBS, and then $1 \mathrm{ml} \mathrm{TRIzol}{ }^{\mathrm{TM}}$ (Takara Biotechnology Co., Ltd., Dalian, China) was added and left for $5 \mathrm{~min}$. The cells were then collected into a 1.5-ml Eppendorf tube, $200 \mathrm{ml}$ chloroform was added and the tube was agitated vigorously and left for $3 \mathrm{~min}$. The tubes were then centrifuged at $15,000 \mathrm{x}$ g for $15 \mathrm{~min}$, and $0.5 \mathrm{ml}$ isopropanol was added into the supernatant and mixed well. The tubes were left at room temperature for $10 \mathrm{~min}$ and then re-centrifuged at $15,000 \mathrm{x} \mathrm{g}$ at $4^{\circ} \mathrm{C}$ for $15 \mathrm{~min}$. Following centrifugation, the supernatant was discarded, and the pellet was washed with $75 \%$ ethanol and finally centrifuged at $15,000 \mathrm{x}$ g at $4^{\circ} \mathrm{C}$ for $15 \mathrm{~min}$. The supernatant was removed and the pellet was dried at room temperature for $15 \mathrm{~min}$. Diethylpyrocarbonate was added to dissolve the pellet and the mRNA was obtained. The contents were then analyzed.

mRNA was reverse transcribed into cDNA according to the instructions in the kits for reverse transcription (Takara Biotechnology Co., Ltd.). The specific procedure was as below: mRNA was quantified, and then random primer, the reverse transcription enzyme, oligo dT and $1 \mathrm{mg}$ mRNA provided in the kits were added, prior to cDNA being obtained by reverse transcription at $37^{\circ} \mathrm{C}$ for $15 \mathrm{~min}$. PCR was performed quantitatively using the real-time fluorescent PCR kit (Takara Biotechnology Co., Ltd.) with the following cycle conditions: $94^{\circ} \mathrm{C}$ for $5 \mathrm{~min}$ and 43 cycles; $94^{\circ} \mathrm{C}$ for $3 \mathrm{sec} ; 63^{\circ} \mathrm{C}$ for $10 \mathrm{sec}$; and $72^{\circ} \mathrm{C}$ for 60 sec. The primers (Sangon Biotech Co., Ltd., Shanghai, China) were as follows: Mouse GAPDH forward sequence, 5'-AGGTCGGTGTGAACGGATTTG-3' and reverse sequence, 5'-GGGGTCGTTGATGGCAACA-3'; mouse OSCAR forward sequence, 5'-CCTAGCCTCATACCCCCAG-3' and reverse sequence, 5'-CGTTGATCCCAGGAGTCACAA-3'; mouse TRAP forward sequence, 5'-CACTCCCACCCTGAGATT TGT-3' and reverse sequence, 5'-CCCCAGAGACATGATGAA GTCA-3'; mouse calcitonin receptor forward sequence, 5'-GAG GTTCCTTCTCGTGAACAG-3' and reverse sequence, 5'-AGT CAGTGAGATTGGTAGGAGC-3'.

Luciferase reporter gene assay. RAW264.7 cells were seeded onto a 24-well plate and cultured until cells were $60-80 \%$ confluent. The culture medium was changed into medium without serum and antibiotics. Lipofectamine ${ }^{\circledR} 2000$ (Invitrogen Life Technologies, Carlsbad, CA, USA), luciferase reporter 
A

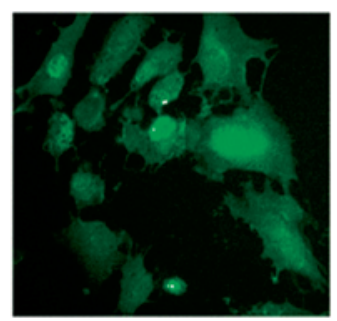

DMSO

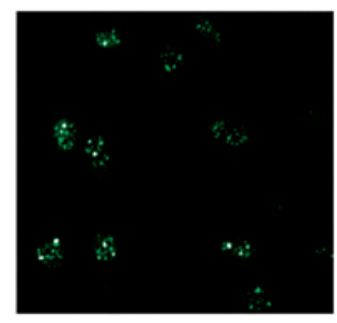

RANKL

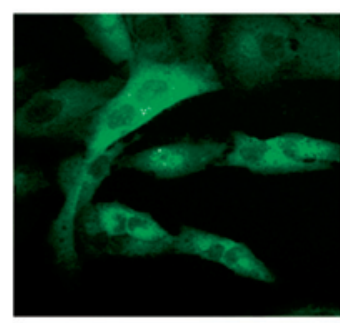

RANKL+Wogonin
B

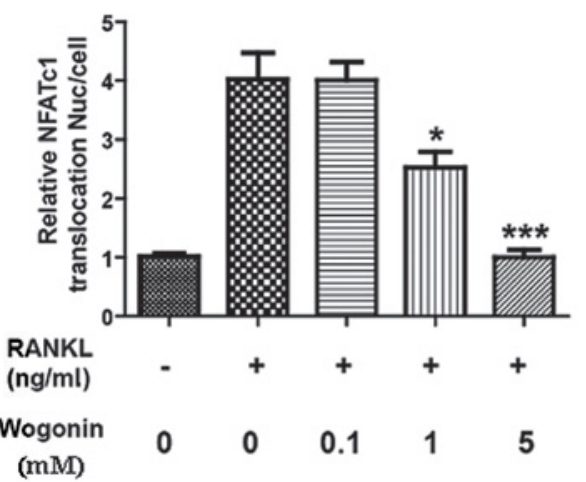

C

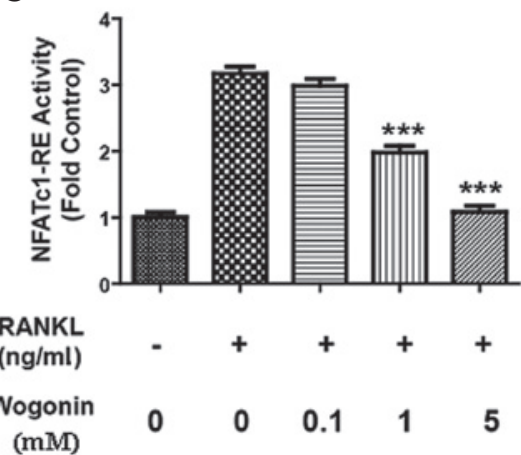

Figure 1. Effect of Wogonin on the translocation and transcriptional activity of NFATc1. U2OS cells stably transfected with EGFP-NFATc1, RANKL $(100 \mathrm{ng} / \mathrm{ml})$ and different concentrations of Wogonin $(5.0,1.0,0.1$ and $0.0 \mathrm{mM})$ were added and incubated for $24 \mathrm{~h}$. The IN Cell ${ }^{\mathrm{TM}}$ Analyzer 1000 was used to observe the translocation of NFATc1. (A) DMSO was used as a control. RANKL (100 ng/ml) significantly stimulated the translocation of NFATc1; Wogonin $(5 \mathrm{mM})$ significantly decreased the translocation of NFATc1 induced by RANKL (magnification, x200). (B) Statistics showed that Wogonin could decrease the translocation of NFATc1 into the nucleus induced by RANKL in a concentration-dependent manner. RAW264.7 cells were transfected with pGL3-promoter-NFATc1-RE, and then RANKL $(100 \mathrm{ng} / \mathrm{ml})$ and different concentrations of Wogonin $(5.0,1.0,0.1$ and $0.0 \mathrm{mM})$ were added and incubated for $24 \mathrm{~h}$. (C) RANKL significantly increased the transcriptional activity of NFATc1 and Wogonin decreased the transcriptional activity of NFATc1 induced by RANKL in a concentration-dependent manner. $n=3 ;{ }^{*} \mathrm{P}<0.05$ and ${ }^{* * *} \mathrm{P}<0.001$ compared with the RANKL group. NAFTc1, nuclear factor of activated T cells c1; EGFP, enhanced green fluorescent protein; DMSO, dimethylsulfoxide, RANKL, receptor activator of nuclear factor $\kappa \mathrm{B}$ ligand; RE, response element.

gene plasmid pRL-SV40 (Promega Corp., Madison, WI, USA) and pGL3-promoter-NFATc1-response element (RE), which was constructed by inserting the NFATc1 cis-acting element sequence (5'-CGCCCAAAGAGGAAAATTTGT TTCATA-3') into the pGL3-promoter plasmid, were prepared using Opti-MEM ${ }^{\circledR}$, kept at room temperature for 5 min and then mixed well, prior to being kept at room temperature for a further $20 \mathrm{~min}$, added into the medium and mixed well. This medium was used to culture the cells for $6 \mathrm{~h}$. The medium was subsequently changed to the complete medium, and RANKL $(100 \mathrm{ng} / \mathrm{ml})$ and different concentrations of Wogonin (5.0, 1.0, 0.1 and $0.0 \mathrm{mM}$ ) were added. DMSO was added as a control and the cells were incubated for $24 \mathrm{~h}$. The lysis buffer provided in the luciferase reporter gene assay kit (Promega Corp.) was used to lyse the cells, and firefly and Renilla luciferase activity was measured using the kit.

Statistical analysis. The results were analyzed using SPSS 17.0 software (SPSS Inc., Chicago, IL, USA), and are expressed as the mean \pm standard deviation. Comparisons between groups were performed using single-factor analysis of variance; $\mathrm{P}<0.05$ was considered to indicate a statistically significant difference.

\section{Results}

Wogonin inhibits the transcriptional activity of NFATcl by inhibiting its translocation into the nucleus. Research has shown that the transcriptional activity of NFATc1 is important for osteoclast differentiation. In normal conditions, NFATc1 is distributed uniformly in the cytoplasm and lacks the ability to activate transcription; however, upon its translocation into the nucleus, NFATc1 gains the ability to activate transcription (11). The specificity of NFATc1 activation can induce osteoclast precursors to differentiate into mature osteoclasts; thus, the inhibition of NFATc1 activation could inhibit osteoclast differentiation and represent a treatment for osteoporosis (12). Based on the literature, a high-throughout screening system for measuring the translocation of NFATc1 into the nucleus was therefore established in the present study. U2OS cells were stably transcribed with EGFP-NFATc1 (U2OS-EGFP-NFATc1); RANKL was added as a stimulus, and natural products (130 single and small molecules in total) were added for screening. The translocation of NFATc1 was observed using the IN Cell Analyzer 1000 (13). RANKL could significantly promote the translocation of NFATc1, while the natural product Wogonin could inhibit the RANKL-induced translocation of NFATc1 in a concentration-dependent manner (Fig. 1A and B). These results showed that Wogonin could inhibit the transcriptional activation activity of NFATc1 by inhibiting the translocation of NFATc1 into the nucleus; therefore, a luciferase reporter gene assay was used to further confirm the effect of Wogonin on the transcriptional activity of NFATc1. Since mouse mononuclear macrophage (RAW264.7) cells can differentiate into osteoclasts following 


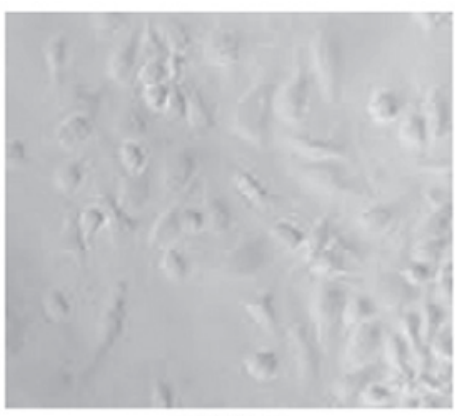

DMSO

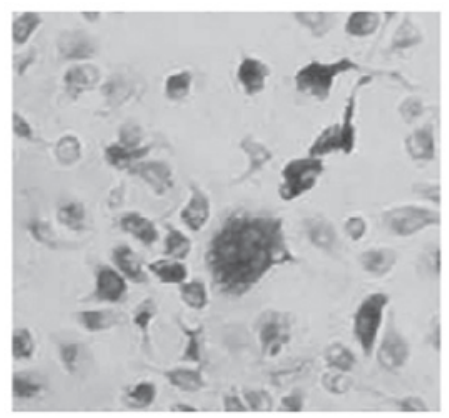

RANKL ( $100 \mathrm{ng} / \mathrm{ml}$ )

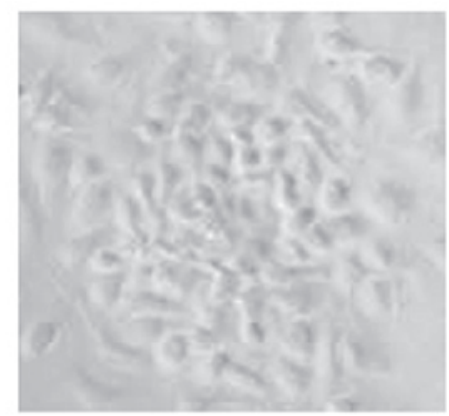

RANKL ( $100 \mathrm{ng} / \mathrm{ml}$ )+ Wogonin $(5 \mu \mathrm{M})$

Figure 2. Wogonin inhibits osteoclast differentiation. A total of $100 \mathrm{ng} / \mathrm{ml}$ RANKL was added to RAW264.7 cells to stimulate differentiation for 3 days. The cells were then stimulated with $100 \mathrm{ng} / \mathrm{ml}$ RANKL and $5 \mathrm{mM}$ Wogonin for 2 days. A tartrate-resistant acid phosphatase detection kit was used to detect the degree of RAW264.7 cell differentiation into osteoclasts. RANKL could significantly stimulate RAW264.7 cell differentiation into osteoclasts but Wogonin (5 mM) could significantly inhibit osteoclast differentiation. Tartrate-resistant acid phosphatase staining (magnification, x200). DMSO, dimethylsulfoxide, RANKL, receptor activator of nuclear factor $\kappa \mathrm{B}$ ligand.

A
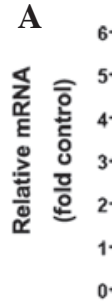

RANKL
(ng/ml)
Wogonin

Wogonin
TRAP

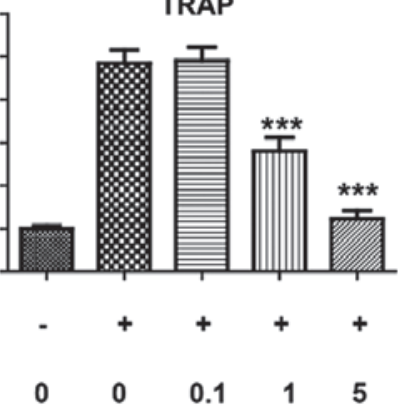

B

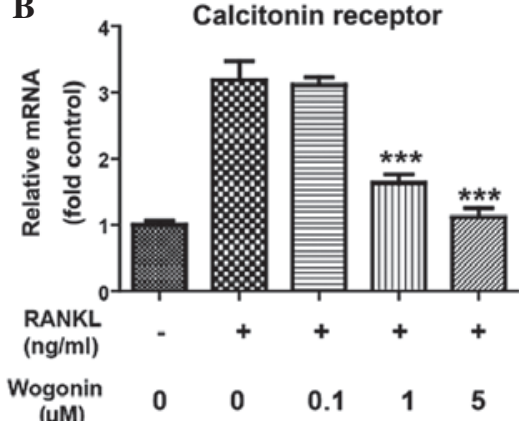

C

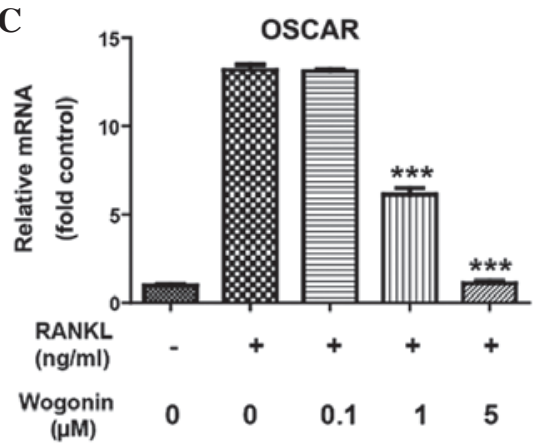

Figure 3. Wogonin inhibits the transcription of important proteins for osteoclasts differentiation. RAW264.7 cells were stimulated with 100 ng/ml RANKL and different concentrations of Wogonin (5.0, 1.0, 0.1 and $0.0 \mathrm{mM}$ ) and incubated for $24 \mathrm{~h}$. The reverse transcription-polymerase chain reaction was used to detect the effect of Wogonin on the mRNA levels of TRAP, calcitonin receptor and OSCAR. RANKL could increase the mRNA level of (A) TRAP, (B) calcitonin receptor and (C) OSCAR in the RAW264.7 cells to different extents, while Wogonin could inhibit the RANKL-induced expression of these mRNAs in a concentration-dependent manner. $n=3 ;{ }^{* * *} \mathrm{P}<0.001$ compared with the RANKL group. RANKL, receptor activator of nuclear factor $\kappa \mathrm{B}$ ligand; TRAP, tartrate-resistant acid phosphatase; OSCAR, osteoclast-associated immunoglobulin-like receptor.

stimulation by RANKL, RAW264.7 cells were transfected with pGL3-promoter-NFATc1-RE plasmid, and then RANKL (100 ng/ml) and different concentrations of Wogonin (5.0, $1.0,0.1$ and $0.0 \mathrm{mM}$ ) were incubated for $24 \mathrm{~h}$. The activity of NFATc1-RE was subsequently measured. Wogonin could inhibit the activation of NFATc1 on its cis-acting element (NFATc1-RE) in a concentration-dependent manner, suggesting that Wogonin could inhibit the transcriptional activity of NFATc1 by decreasing its translocation into the nucleus (Fig. 1C).

Wogonin inhibits osteoclast differentiation. Since it was shown that Wogonin could significantly inhibit the translocation of NFATc1 into the nucleus and its transcriptional activity, and that inhibiting the transcriptional activity of NFATc1 could inhibit osteoclast differentiation, mouse mononuclear macrophage (RAW264.7) cells were stimulated by RANKL to induce osteoclast differentiation, and the effect of Wogonin on the RAW264.7 cell differentiation into osteoclasts was assessed. Wogonin could significantly inhibit the RAW264.7 cell differentiation into osteoclasts, sugge sting that Wogonin could inhibit osteoclast differentiation and thus represent a potential therapeutic agent for treating osteoporosis (Fig. 2).

Wogonin inhibits osteoclast differentiation by inhibiting the transcription activity of NFATc1. NFATc1 plays a vital role in osteoclast differentiation. Following its translocation into the nucleus and its stimulation of transcriptional activity, NFATc1 can enhance multiple stimuli or activate the transcription of proteins that are important for osteoclasts, such as TRAP, OSCAR and calcitonin receptor, thus facilitating osteoclast differentiation. qPCR was therefore used in the present study to detect the effect of Wogonin on the transcriptional activity of TRAP, OSCAR and calcitonin receptor in RAW264.7 cells, and thus further clarify whether Wogonin could inhibit osteoclast differentiation by inhibiting the translocation of NFATc1 into the nucleus. As shown in Fig. 3, RANKL could increase the mRNA level of TRAP, calcitonin receptor and OSCAR in the RAW264.7 cells to different extents, suggesting that Wogonin could inhibit transcription of important proteins for osteoclast differentiation and thus further confirming that Wogonin could inhibit osteoclast differentiation by inhibiting the transcriptional activity of NFATc1. 


\section{Discussion}

Osteoporosis is a systemic bone disease associated with age. The symptoms are a decrease in bone mass and density, leading to pain and fractures. Currently, the clinical drugs for treatment can be classified into two main categories: Calcium preparations, such as calcium gluconate and vitamin D, which supplement the calcium content in the body, and agents that inhibit bone absorption and facilitate bone formation, such as bisphosphonates. However, calcium preparations can only improve the relevant symptoms of osteoporosis, and bisphosphonates have toxic adverse effects; therefore, a novel drug for osteoporosis would be valuable.

NFATc1 is an important factor involved in the inhibition of osteoclast differentiation. NFATc1 can translocate into the nucleus and upregulate the transcription of several proteins, such as TRAP and OSCAR, which are important for osteoclast differentiation, following stimulation by RANKL. Anti-osteoporosis drugs targeting NFATc1 are therefore important for osteoporosis treatment $(14,15)$. The present study identified the natural product Wogonin through a high-throughout screening system. Wogonin could significantly inhibit the translocation of NFATc1 into the nucleus and thus inhibit its transcriptional activity. In further investigations, it was found that Wogonin could significantly decrease the mRNA expression level of TRAP, OSCAR and calcitonin receptors; these mRNAs are regulated by NFATc1 and play a vital role in osteoclast differentiation. Furthermore, Wogonin could inhibit the RANKL-induced mouse mononuclear macrophage differentiation into osteoclasts. In combination, these results showed that Wogonin could inhibit osteoclast differentiation by inhibiting the transcriptional activity of NFATc1, and thus suggested that Wogonin could have a therapeutic role in osteoporosis.

Wogonin has a wide range of pharmacological activities and exerts anti-cancer and anti-inflammatory effects. Numerous reports have described the role of Wogonin in anti-cancer treatments $(16,17)$, detailing its significant growth inhibitory effect on tumor cells $\left(\mathrm{IC}_{50}, 15-200 \mathrm{mM}\right)$. In addition, in vivo studies have demonstrated its anti-tumor activity $(18,19)$. Mice were intravenously administered $40 \mathrm{mg} / \mathrm{kg}$ Wogonin for 7 days and the inhibition rate for the growth of S180 transplanted tumors was 53\% (18), while an intraperitoneal injection of $200 \mathrm{mg} / \mathrm{kg}$ Wogonin could completely inhibit leukemia and CEM cells (19). The anti-cancer mechanisms of Wogonin consist of multiple aspects, such as inducing apoptosis and differentiation, affecting the cell cycle, inhibiting tumor angiogenesis and inhibiting telomerase $(20,21)$.

In conclusion, the present study has shown that Wogonin may inhibit osteoclast differentiation by inhibiting the translocation of NFATc1 into the cell nucleus and represents an effective small molecule for further study into anti-osteoporosis drugs. Wogonin also provides a novel mechanism for the Chinese medicine Scutellaria baicalensis in the treatment of osteoporosis.

\section{References}

1. Zaidi M: Skeletal remodeling in health and disease. Nat Med 13: 791-801, 2007.

2. Novack DV and Teitelbaum SL: The osteoclast: Friend or foe? Annu Rev Pathol 3: 457-484, 2008.

3. Horsley V, Aliprantis AO, Polak L, Glimcher LH and Fuchs E: NFATc1 balances quiescence and proliferation of skin stem cells. Cell 132: 299-310, 2008.

4. Pei J, Li B, Gao et al: Fluoride decreased osteoblastic bone resorption through the inhibition of NFATc1 gene expression. Environ Toxicol 29: 588-595, 2014.

5. Negishi-Koga T and Takayanagi $\mathrm{H}: \mathrm{Ca}^{2+}-\mathrm{NFATc} 1$ signaling is an essential axis of osteoclast differentiation. Immunol Rev 231: 241-256, 2009.

6. Takayanagi H, Kim S, Koga T, et al: Induction and activation of the transcription factor NFATc1 (NFAT2) integrate RANKL signaling in terminal differentiation of osteoclasts. Dev Cell 3: 889-901, 2002.

7. Kim K, Kim JH, Lee J, et al: Nuclear factor of activated T cells c1 induces osteoclast-associated receptor gene expression during tumornecrosis factor-related activation-induced cy tokine-mediated osteoclastogenesis. J Biol Chem 280: 35209-35216, 2005.

8. Kim Y, Sato K, Asagiri M, Morita I, Soma K and Takayanagi H: Contribution of nuclear factor of activated $T$ cells $\mathrm{cl}$ to the transcriptional control of immunoreceptor osteoclast-associated receptor but not triggering receptor expressed by myeloid cells- 2 during osteoclastogenesis. J Biol Chem 280: 32905-32913, 2005.

9. He L, Lu N, Dai Q, et al: Wogonin induced G1 cell cycle arrest by regulating Wnt/ $\beta$-catenin signaling pathway and inactivating CDK8 in human colorectal cancer carcinoma cells. Toxicology 312 : 36-47, 2013

10. Chen XM, Bai Y,Zhong YJ, et al: Wogonin has multiple anti-cancer effects by regulating c-Myc/SKP2/Fbw $7 \alpha$ and $\mathrm{HDAC} 1 / \mathrm{HDAC} 2$ pathways and inducing apoptosis in human lung adenocarcinoma cell line A549. PLoS One 8: e79201, 2013.

11. Masuda ES, Imamura R, Amasaki Y, Arai K and Arai N: Signalling into the T-cell nucleus: NFAT regulation. Cell Signal 10: 599-611, 1998.

12. Collin-Osdoby P and Osdoby P: RANKL-mediated osteoclast formation from murine RAW 264.7 cells. Methods Mol Biol 816: 187-202, 2012.

13. Kim SJ, Ding W, Albrecht B, Green PL and Lairmore MD: A conserved calcineurin-binding motif in human $\mathrm{T}$ lymphotropic virus type $1 \mathrm{p} 12 \mathrm{I}$ functions to modulate nuclear factor of activated T cell activation. J Biol Chem 278: 15550-15557, 2003.

14. Ikeda F, Nishimura R, Matsubara T, et al: Critical roles of c-Jun signaling in regulation of NFAT family and RANKL-regulated osteoclast differentiation. J Clin Invest 114: 475-484, 2004.

15. Ikeda F, Nishimura R, Matsubara T, Hata K, Reddy SV and Yoneda T: Activation of NFAT signal in vivo leads to osteopenia associated with increased osteoclastogenesis and bone-resorbing activity. J Immunol 177: 2384-2390, 2006.

16. Xu M, Lu N, Zhang H, et al: Wogonin induced cytotoxicity in human hepatocellular carcinoma cells by activation of unfolded protein response and inactivation of AKT. Hepatol Res 43: 890-905, 2013.

17. Chow SE, Chen YW, Liang CA, Huang YK and Wang JS: Wogonin induces cross-regulation between autophagy and apoptosis via a variety of Akt pathway in human nasopharyngeal carcinoma cells. J Cell Biochem 113: 3476-3485, 2012.

18. Wang W, Guo QL, You QD, et al: The anticancer activities of wogonin in murine sarcoma S180 both in vitro and in vivo. Biol Pharm Bull 29: 1132-1137, 2006.

19. Zhang HW, Yang Y, Zhang K, et al: Wogonin induced differentiation and G1 phase arrest of human U-937 leukemia cells via PKCD phosphorylation. Eur J Pharmacol 591: 7-12, 2008.

20. Baumann S, Fas SC, Giaisi M, et al: Wogonin preferentially kills malignant lymphocytes and suppresses T-cell tumor growth by inducing PLCC1-and $\mathrm{Ca}^{2+}$-dependent apoptosis. Blood 111: 2354-2363, 2008.

21. Tsai CF, Yeh WL, Huang SM, Tan TW and Lu DY: Wogonin induces reactive oxygen species production and cell apoptosis in human glioma cancer cells. Int J Mol Sci 13: 9877-9892, 2012. 